\title{
Growth and forms of Laplacian aggregates
}

\author{
A. Sánchez \\ Escuela Politécnica Superior, Universidad Carlos III, Avenida del Mediterráneo 20, 28913 Leganés, Madrid, Spain, \\ and Theoretical Division and Center for Nonlinear Studies, Los Alamos National Laboratory, \\ Los Alamos, New Mexico 87545 \\ F. Guinea* and L. M. Sander \\ The Harrison M. Randall Laboratory of Physics, The University of Michigan, Ann Arbor, Michigan 48109-1120 \\ V. Hakim \\ Laboratoire de Physique Statistique, Ecole Normale Superiéure, 24 Rue Lhomond, 75231 Paris CEDEX 05, France \\ E. Louis \\ Departamento de Física Aplicada, Universidad de Alicante, Apartado 99, 09080 Alicante, Spain
}

(Received 10 February 1993)

\begin{abstract}
The shapes and general morphological properties of aggregates grown following the $\eta$ rule $\left(v_{\text {surface }} \propto|\mathbf{E}|^{\eta}\right)$ [L. Niemeyer, L. Pietronero, and H. J. Wiessmann, Phys. Rev. Lett. 52, 1033 (1984)] have been investigated. $v_{\text {surface }}$ is the velocity at the interface and $\mathbf{E}$ the electric field. The fractal dimension decreases monotonically from its diffusion-limited aggregation value $(\eta=1)$ to a number indistinguishable from 1 at $\eta \gtrsim 4$. Simultaneously, the multifractal properties become independent of $\eta$. An alternative method to generate large clusters, and gain insight into the growth process, is also presented. Various analytical approximations are discussed.
\end{abstract}

PACS number(s): 82.20.Wt, 75.10.Jm, 75.10.Lp, 75.30.Ds

\section{INTRODUCTION}

Growth out of equilibrium is a fascinating, although poorly understood, topic. Extensive numerical work has revealed many features of some of the most widespread growth phenomena in nature.

A good understanding exists, at the phenomenological level, of diffusion-limited aggregation (DLA) [1], which in turn describes many other pattern-formation situations. Moreover, it is deeply connected to solidification. It is known that the rich, fractal structure of DLA aggregates arises from the tip-splitting instability which is inferred from numerical and analytical calculations. Phenomenological schemes reproduce some of the most conspicuous global features, like the fractal dimension $[2,3]$.

A comprehensive theoretical understanding is, however, lacking. The difficulty of the problem has lead us to analyze, in a systematic way, related growth processes where the complexity of the resulting patterns is much smaller. We focus on the $\eta$ model, which follows the same growth rules as DLA [5], except for the fact that the velocity at the interface depends on the field in a different way, $v \propto|\mathbf{E}|^{\eta}$. As such, this model is a reasonable approximation to the phenomenon of dielectric breakdown. The resulting aggregates for large values of $\eta$ are simpler than DLA patterns (which corresponds to $\eta=1$ ) [4]. An even simpler approximation, the Laplacian random walk (LRW), has been introduced [6,7]. It is worth noting that even this simple model shows a nontrivial fractal structure [8].
In Sec. II we present our method of generation of aggregates, and show typical examples. Section III discusses the multifractal spectra of the growth-site probability density (GSPD) of these aggregates [9]. In Sec. IV we present an alternative method of generating aggregates, which allows for the formation of larger systems, and gives some additional insight into the nature of the growth. A discussion of the main results is given in Sec. V. Finally, conclusions, open questions and prospects for the future are given in Sec. VI.

In a previous work [10] the intriguing possibility that the $\eta$ model effectively reduced to Laplacian walks above a critical $\eta$ was suggested and preliminary tests were performed. This idea was motivated by recent analytical findings [11] on simplified growth models. In the present work, we further investigate this possibility. Previous studies of this model have been reported on in [5, 12] for the range $\eta \leq 2$, in [4] for $\eta \leq 5$ and very many small clusters, and very recently in [13] for $\eta \leq 1$. Interestingly, this last work provides evidence for another transition in the morphology of the aggregates.

\section{METHOD}

The main obstacle in the study of the $\eta$ growth model has always been the generation of large aggregates. The difficulty arises from the fact that, although the model can be (at least for integer $\eta$ ) formulated in terms of random walks [12], much in the same way as DLA, simula- 
tions become prohibitively time consuming for $\eta \geq 2$. On the other hand, as the value of $\mathbf{E}$ depends on a Laplacian field defined throughout the space between the growing aggregate and some external boundary, a time-consuming calculation is required after each growth event. Moreover, for high values of $\eta$, the resulting patterns are very dilute, and soon reach the boundary.

In the present work, we solve Laplace's equation in hexagonal lattices of different sizes (outer hexagon sides 128,250 , and 500 , or, equivalently lattice sizes $256 \times 256$, $500 \times 500$, and $1000 \times 1000)$ to exclude possible finite-size effects. The choice of the hexagonal lattice was made to minimize anisotropy effects. Unlike the square lattice, the continuum limit of the discrete Laplace equation is isotropic up to sixth derivative terms. Typical aggregates for high $\eta$ 's contain 300-400 particles for outer hexagon side 250 , and $600-700$ for outer hexagon side 500 . For $\eta=1$ we can generate patterns with up to 20000 particles (for this value we did not study the largest lattice). An alternative method, which can be used to obtain even larger aggregates, is discussed in Sec. IV.

After the growth process has finished, aggregates are characterized by means of several parameters, most remarkably two: the fractal dimension and the multifractal spectrum of the GSPD. As for the first one, two different definitions of fractal dimension have been used, namely, standard mass-radius scaling and box counting. As the former turned out to be much less precise than the latter, we eventually chose box counting as our fractaldimension-measurement algorithm. Our second indicator is the multifractal spectrum of the GSPD, which we define, following [9], as the probability that a perimeter site becomes part of the cluster in the next growth step. We again calculate this characteristic function, $f(\alpha)$, in two ways: by Legendre transforming the box-counting generalized dimensions $D_{q}[14]$, and by the direct procedure proposed by Chhabra and Jensen [15]. The reason for using both methods is the great inaccuracy of the Legendretransform algorithm in the negative- $q$ (right- $\alpha$ side) region of the spectrum. The so-obtained results showed an extremely good agreement, which gives us confidence in our spectrum calculations. Satisfactory comparison to previous results $[4,16]$ further supports our conclusions. Other auxiliary parameters we considered are the ratio of the number of particles in the aggregate to the diameter of the lattice (two times the outer hexagon side), and the ratio of the number of particles in the aggregate to the number of particles in its perimeter. Finally, it is important to realize that the singularities of the patterns are also related to the noise which is generated during the growth process, which some of us have also studied [17]. The present results are consistent, and improve the results reported in [17].

To conclude this section, some comments are in order on how we obtain our results and what is the subsequent error. The values of $\eta$ studied range from 0 to 10 , in increments of 1 (we took 0.5 when analyzing the most interesting regions). The results are averaged over at least three aggregates for each $\eta$ value and each size. For $\eta=$ 4,6 , and 8 we checked our results taking averages over 15 aggregates. The error bars in the results are calculated from the square root of the sum of squares of the statistical spread between different realizations, the inherent limitations of the box-counting method, and the errors of the linear regressions required in all the computations. Even with all these limitations, we get accuracies better than a few percent. It seems very difficult to us to get much better results, which are needed in order to find a possible sharp transition and to characterize it, without using an exceedingly greater amount of computer time.

\section{NUMERICAL RESULTS}

In Fig. 1 we present typical aggregates for $\eta=$ $1,2,4,6,8$, and 10 . From the general shape of these aggregates, we can distinguish two different regimes: patterns which show a proliferation of tip splittings $(\eta \lesssim 4)$, and patterns which show no tip splitting, and which look like a thin ribbon $(\eta \gtrsim 4)$. The latter are almost indistinguishable from LRW's $[6,7]$. The main difference comes from the presence, in our systems, of a small number of short sidebranches scattered along the aggregate.

The corresponding singularity spectra are shown in Fig. 2. These $f(\alpha)$ curves show various characteristic behaviors:

(i) For $\eta=1$, there is a broad tail for large values of $\alpha$. It means a large number of very weak singularities, which arise from regions of the aggregate where screening is important.

(ii) This tail disappears for $\alpha \gtrsim 2$.

(iii) Finally, when we reach the region $\eta \gtrsim 4$, the function $f(\alpha)$ changes little, if at all. Its shape is much narrower than in the previous regimes, meaning that the shapes are well described by a few parameters. However, it retains a finite width, signalling that some multifractal character remains. This width is significantly larger than our numerical accuracy.

Figure 3 shows the fractal dimension of the aggregates, as function of $\eta$. The curve becomes indistinguishable from 1 for $\eta \geq 6$. Our results do not exclude the possibility of a sharp transition at a finite value of $\eta$. This possibility is supported by the ratio of the number of particles to hexagon size (see Fig. 4) which becomes less than 1 for $\eta \lesssim 4$, indicating that the cluster fits inside the lattice without deviating from a straight line, and by the ratio of the number of particles to perimeter size, that reaches the value 2 in the same $\eta$ region, a behavior again typical of a branchless structure (see Fig. 5). It is worth noting that an aggregate may have branches of a finite size above the transition to $D_{f}=1$. The fact that the ratio plotted in Fig. 5 goes smoothly to 2 possibly indicates that small branches survive at high values of $\eta$. The average size tends continously to zero as $\eta \rightarrow \infty$.

\section{IV. $\boldsymbol{\eta}$ AGGREGATES AS CHARGED SYSTEMS}

The $\eta$ aggregates can be thought of as charged metallic systems at constant potential. Their inner charge distribution, plus additional charges at the external boundary, give rise to fields which, in turn, determine the growth. For a given shape, represented by a discrete set of sites, 


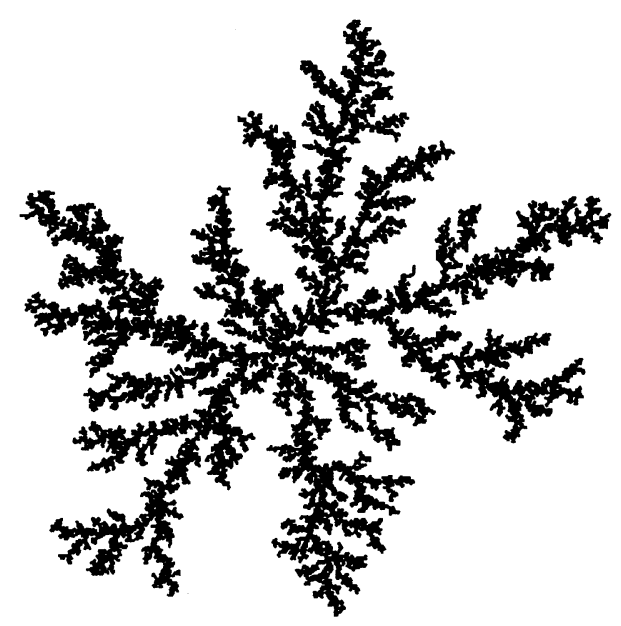

(a)

(b)
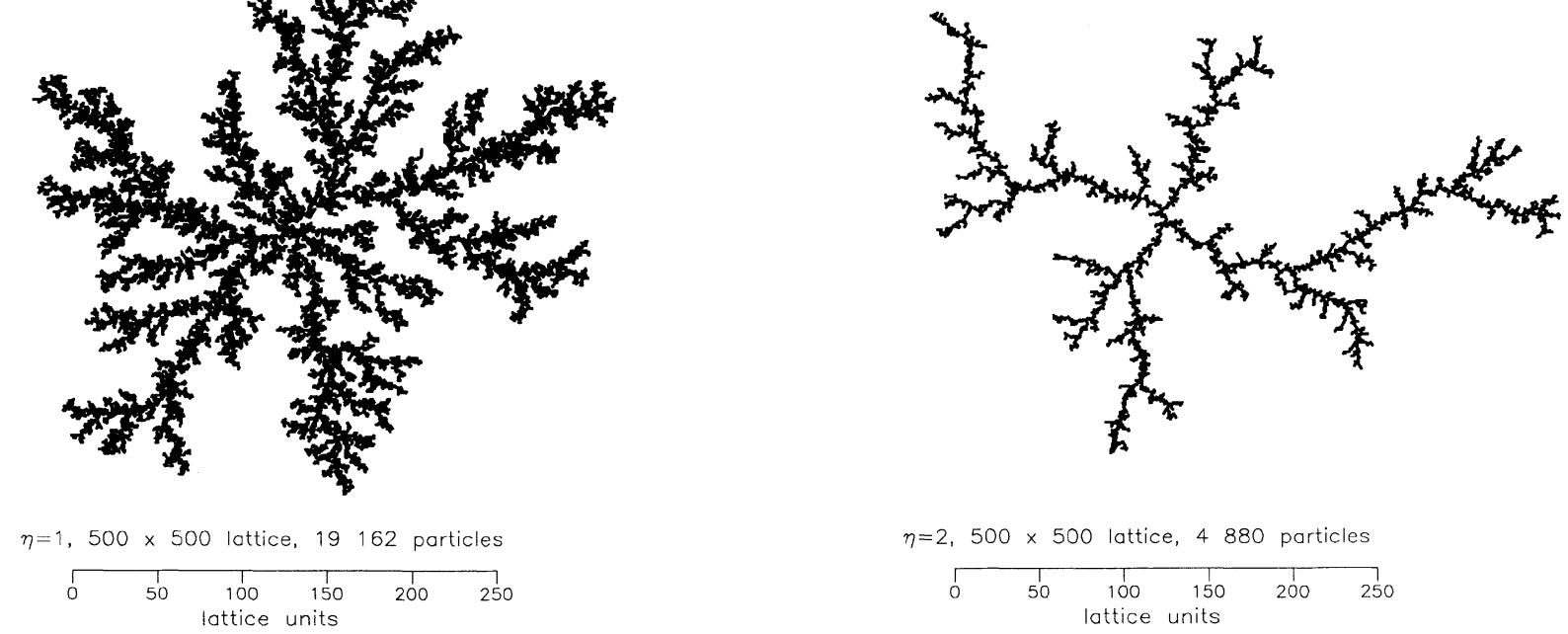

(c)
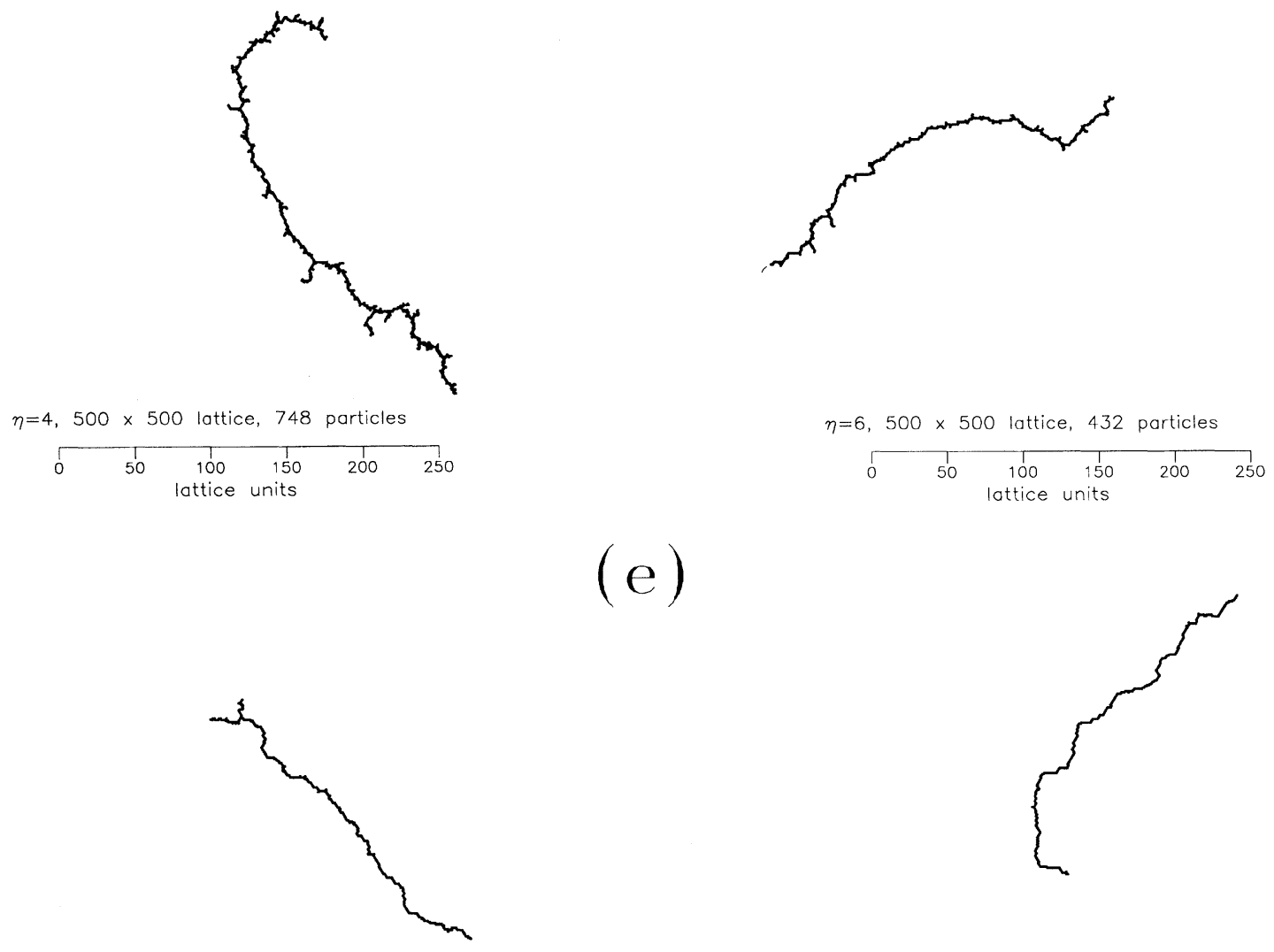

(f)
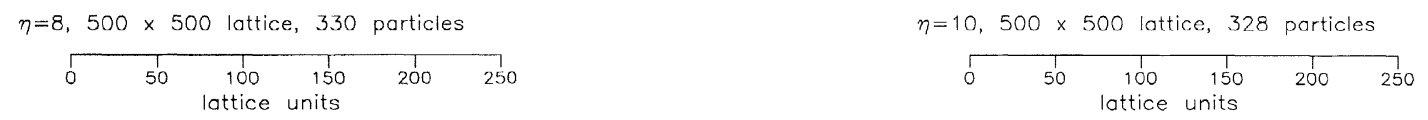

FIG. 1. Typical aggregates obtained for different values of $\eta$. (a) $\eta=1$, (b) $\eta=2$, (c) $\eta=4$, (d) $\eta=6$, (e) $\eta=8$, (f) $\eta=10$. 

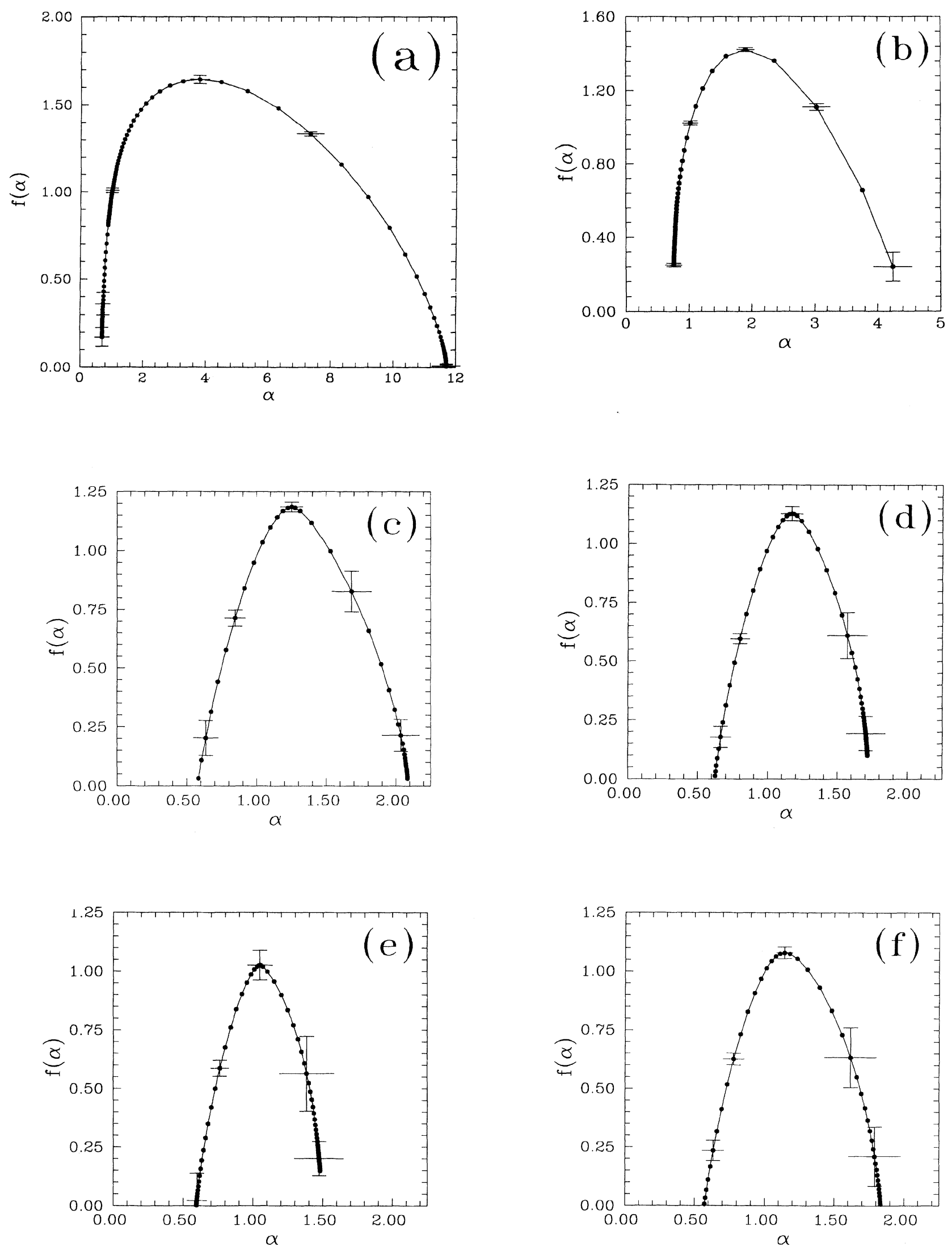

FIG. 2. Function $f(\alpha)$ for different values of $\eta$. (a) $\eta=1$, (b) $\eta=2$, (c) $\eta=4$, (d) $\eta=6$, (e) $\eta=8$, (f) $\eta=10$. 


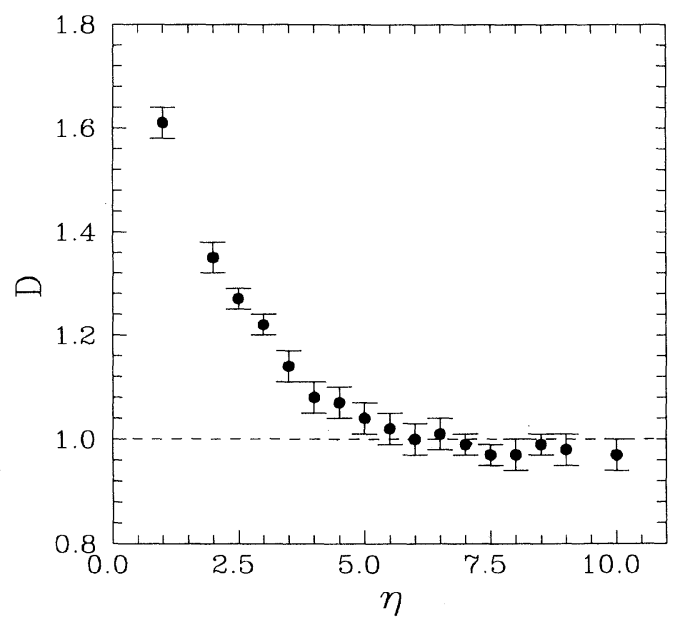

FIG. 3. Fractal dimension $D$ as a function of $\eta$.

the calculation of the charge distribution can be reduced to the inversion of a matrix, with as many entries as sites. This scheme, also referred to as the Green's function method, has been used to determine the charges, fields, and growth probabilities in the $\eta$ and related models. Its main limitation is the impossibility of manipulating large matrices. The maximum sizes achieved in this fashion contain around 500 particles [4].

On the other hand, the charges within the aggregate vary little in regions where screening is important, or far away from tips and wedges. This property has lead us to simplify the problem by assuming that the charge distribution, within the aggregate, is constant within blocks of different sizes. These sizes are adjusted so that the total charge within each block changes little from block to block. Assuming this stepwise constant distribution

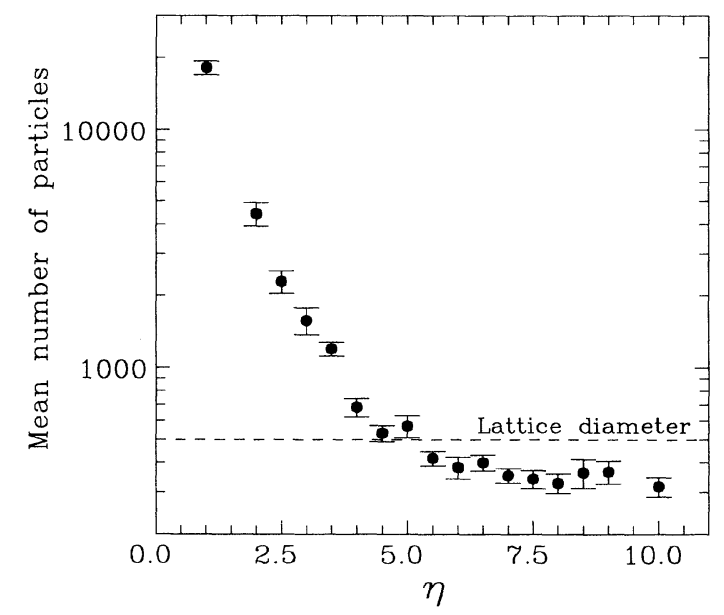

FIG. 4. Mean number of particles in the aggregates as a function of $\eta$. The dashed line shows the diameter of the cell used in the calculations. The lattice diameter is twice the outer hexagon side and is equal to the square lattice side used to parametrize the hexagonal lattice and also to the maximum straight length which fits inside the outer hexagon.

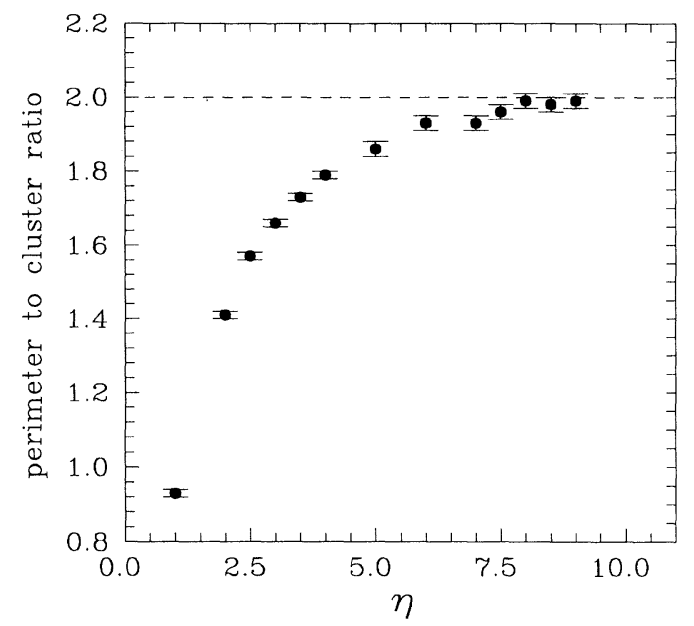

FIG. 5. Mean ratio between the particles at the perimeter of an aggregate and those within it.

of charges, the number of independent variables to be adjusted is equal to the number of blocks. The question of finding the charge distribution which gives rise to a constant potential throughout the aggregate can be formulated as the determination of the charge distribution which minimizes the electrostatic energy, with the total charge fixed. Cast in this way, the problem to be solved is easily constrained to stepwise constant-charge distributions. Once the charges are known, the growth probability can be computed, and new sites added to the aggregate.

The formulation of the growth process in terms of charges internal to the aggregate has the advantage of getting rid of the external electrode, and its associated boundary effects. The limitations of the method can be checked by changing the number of blocks in which the aggregate is divided each time. In our calculations we find good convergence for $\eta \geq 3$ and 300-500 blocks. While this method gives good examples of small DLA clusters $(\eta=1)$, a high numerical accuracy is required to generate large systems with small values of $\eta$. For simplicity, the growth process is defined so that a growing site within the aggregate is chosen, with probability proportional to the charge at that site to the $\eta$ power. Then, all nonoccupied neighboring sites are added to the aggregate. As there is a direct correspondence between the charges at the boundary of the aggregate and the electric field outside it, we think that this is not a serious alteration of the model.

Typical results for aggregates are shown in Fig. 6. The positions and sizes of the blocks used are shown in Fig. 7. In Fig. 8 we show the charge distribution near one of the tips of the growing aggregate. Our simulations do not change the results discussed in the preceding section. The same regimes and shapes, as functions of $\eta$, are recovered. This implies that the outer electrode, used within the standard method, does not influence the results. On the other hand, the change in the way the growth process is implemented also seems to leave the global properties unchanged. 


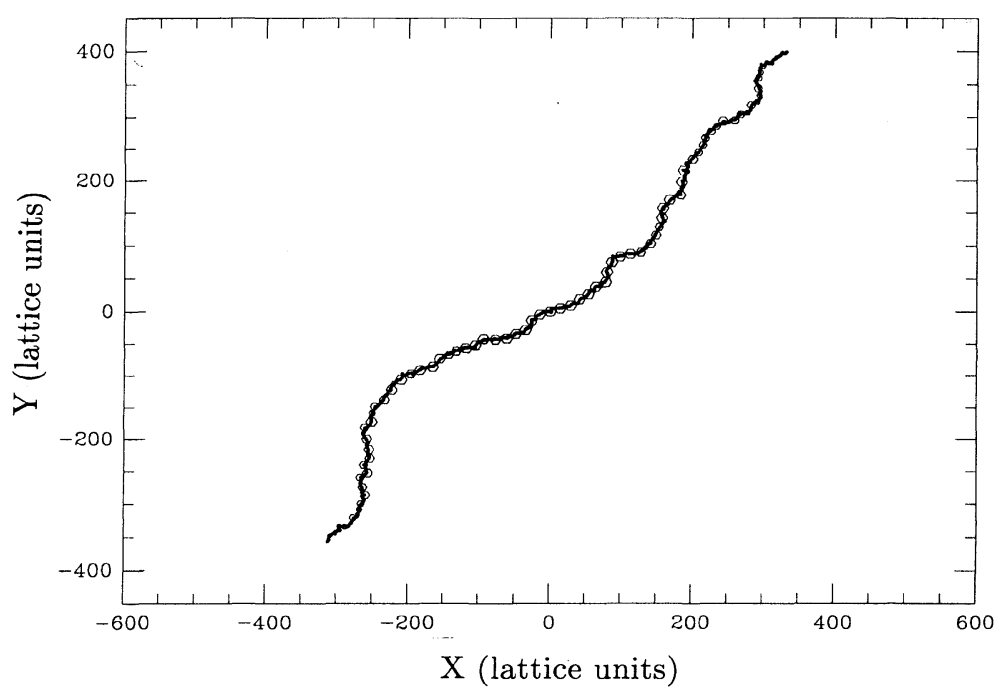

FIG. 6. Typical aggregate obtained using the method described in Sec. IV $(\eta=8)$.

\section{DISCUSSION}

All the results presented previously show that there is a trend towards simpler patterns as the value of $\eta$ is increased. The tip-splitting instability disappears, leading to ribbonlike aggregates. Then, the shapes of these patterns lose structure, becoming more and more similar to the Laplacian random path already discussed. This analogy can be pursued further, by looking at the total growth far from the tip as a function of $\eta$. We can assume that near the tip, the electric field decays as $\sim 1 / \sqrt{\left|\mathbf{R}_{\text {tip }}-\mathbf{R}\right|}$. This is the distribution of charge at the tip of a sharp needle. If the tip is moving, as a first approximation, in a straight line and at constant velocity, the amount of material deposited at a given site is

$$
\begin{aligned}
& M(\mathbf{R}, t)=\int_{\mathbf{R}_{\mathrm{tip}}=\mathbf{R}}^{t}\left|\mathbf{R}_{\mathrm{tip}}(t)-\mathbf{R}\right|^{-\frac{\eta}{2}} d t \\
& \mathbf{R}_{\mathrm{tip}}(t)=\mathbf{R}_{0}+v_{\mathrm{tip}} t
\end{aligned}
$$

The total mass $M(\mathbf{R})$ deposited at all sites between the seed and $\mathbf{R}$, between $t=0$ and $t \rightarrow \infty$, can be calculated by integration over $\mathbf{R}$ and $t$. Its value is finite for $\eta \geq 4$. Thus, we can conclude that a thin ribbon can be the stable shape for high values of $\eta$. Note that the same argument, for $\eta=1$, gives that a point separated by a distance $D$ from the tip, accumulates a mass proportional to $\sqrt{D}$. This result can be seen as a simple approximation to the parabolic shape of a deterministic finger in DLA and dendritic growth.

The analysis in the preceding paragraph suggests that the random walk is a good approximation to the $\eta$ aggregates for $\eta \geq 4$. Thus, we need only to keep track of the propagating tip, whose growth is determined by the electric field generated by the charges distributed throughout the aggregate. Let us assume that the walk, at a given instant, is parametrized by an intrinsic coordinate, the total length $\tau$, and the angle of its motion with respect to a fixed coordinate system, $\theta(\tau)$. Then, the coordinates $X, Y$ of the tip at a given instant are such that

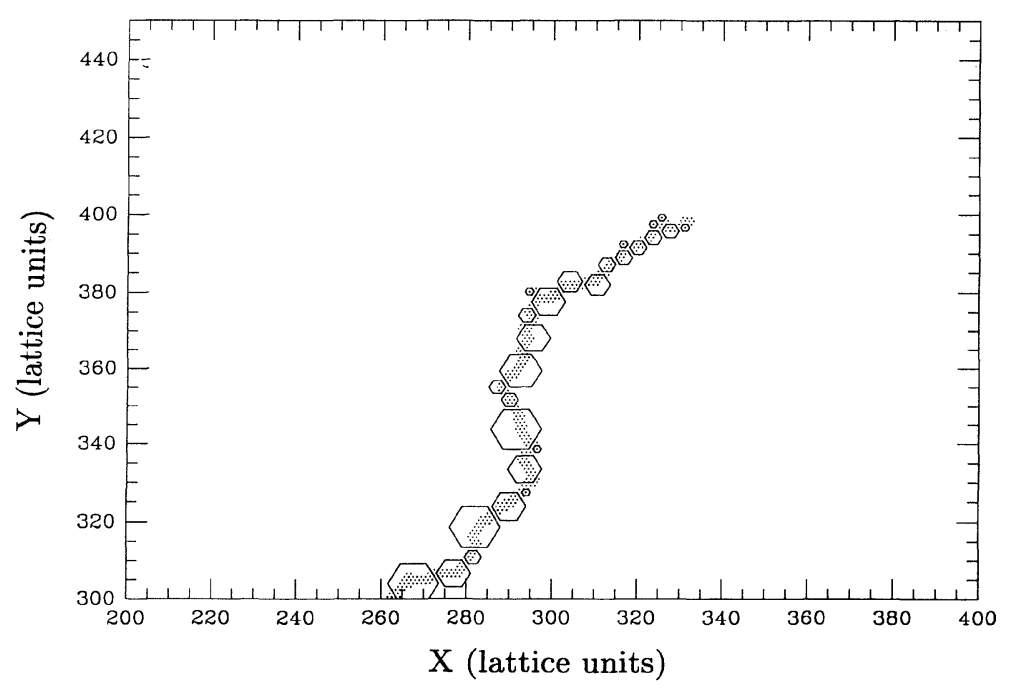

FIG. 7. Blocks generated when dividing an aggregate, for $\eta=8$. The number of points within the aggregate is 6100 . Only the region near one of the tips is shown. 


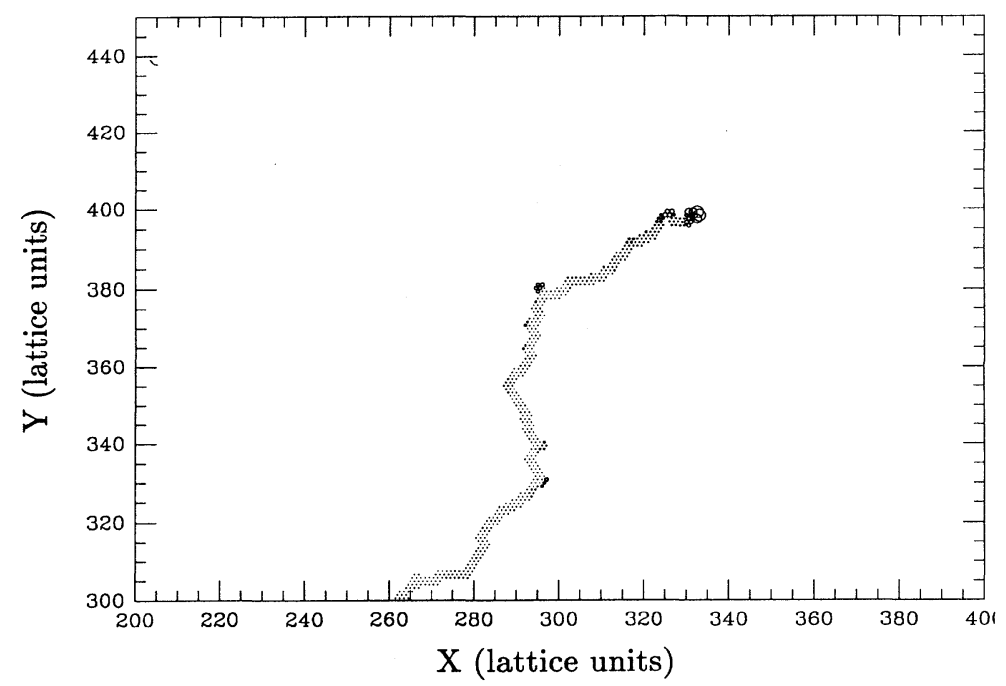

FIG. 8. Charges near the tip of the aggregate shown in Fig. 7 .

$$
\begin{aligned}
& \frac{\partial X}{\partial \tau}=\cos [\theta(\tau)] \\
& \frac{\partial Y}{\partial \tau}=\sin [\theta(\tau)] .
\end{aligned}
$$

The field at the growing tip, assuming that the path starts at $\tau=0$ and ends at $\tau=\tau_{0}$, will be

$$
\begin{aligned}
& E_{x}=\int_{0}^{\tau_{0}} d \tau \rho(\tau) \frac{X\left(\tau_{0}\right)-X(\tau)}{\left[X\left(\tau_{0}\right)-X(\tau)\right]^{2}+\left[Y\left(\tau_{0}\right)-Y(\tau)\right]^{2}}, \\
& E_{y}=\int_{0}^{\tau_{0}} d \tau \rho(\tau) \frac{Y\left(\tau_{0}\right)-Y(\tau)}{\left[X\left(\tau_{0}\right)-X(\tau)\right]^{2}+\left[Y\left(\tau_{0}\right)-Y(\tau)\right]^{2}}
\end{aligned}
$$

with the values of $X(\tau)$ and $Y(\tau)$ given by the integral of Eq. (1). The function $\rho(\tau)$ gives the charge distribution along the path.

A straight needle is given by $\theta(\tau)=$ const. Modifications of this shape will arise when the electric field at the tip will deviate from the direction of growth, defined by $\theta$. If these the deviations are small, we can write

$$
\frac{\partial \theta}{\partial \tau}=k\left[-E_{x} \sin (\theta)+E_{y} \cos (\theta)\right]
$$

where the constant $k$ depends on $\eta$, and tends to a finite value for $\eta \rightarrow \infty$. This value, as well as the general dependence of $k$ on $\eta$, is not universal, and will depend on the short-distance cutoff used to define the charges and fields.

For a straight needle we can analytically compute $\rho(\tau)$. We will assume that the corrections to that value will depend on the local radius of curvature, which is proportional to $(\partial \theta / \partial \tau)^{-1}$. Then, for a path of length $\tau_{0}$, we have

$$
\rho(\tau)=\frac{A}{\sqrt{\tau\left(\tau_{0}-\tau\right)}}+O\left(\frac{\partial \theta}{\partial \tau}\right) .
$$

This set of equations should contain the most relevant physics of a Laplacian random walk, except for the noise. It is easy to see that they always describe a straight needle at large scales. The reason is schematically shown in Fig. 9. The field at the tip of the growing aggregate comes mostly from the region near the tip itself, plus contributions from the rest of the system, most notably the other end and the regions of high curvature. Then, the field will tend to heal any deviation, over long scales, from a straight line between the starting and the final points. The same effect should take place if the two ends are growing simultaneously.

It is interesting to note that, in polar coordinates, and taking the initial point as the origin, the angular coordinate at the tip changes in a monotonic fashion. The angular component of the field has a sign determined by the initial conditions (see Fig. 9), which is conserved during the growth process. Thus, the system, before reaching its steady state, has a tendency to develop spiral patterns. The pitch of the spiral increases with time, so that the shape becomes more straight as the system grows. This effect is clearly distinguishible in many simulations (see Fig. 10), although difficult to characterize quantitatively.

From previous analysis, we infer that the noise plays a crucial part in determining the global shape of the aggregate. We will analyze it using, as in the previous case, the high $\eta$ limit as a starting point.

We assume that, in this limit, the shape of the aggre-

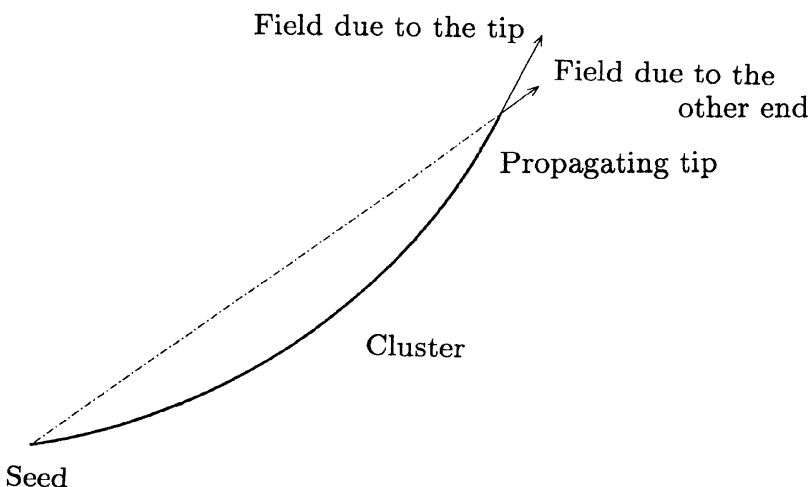

FIG. 9. Schematic view of the forces which determine the growth of an aggregate at high values of $\eta$. See text for details. 

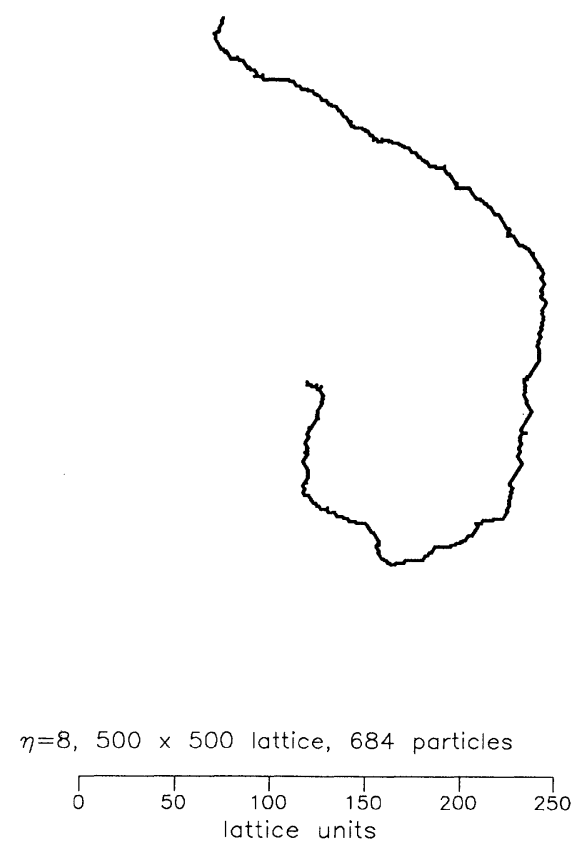

FIG. 10. Spiral aggregate obtained for $\eta=8$.

gate resembles that of a straight needle. It implies that the probability for the tip to move forward is greater than the probability to move sideways or backward. In addition, a straight needle is generated only if the probabilities of moving to the right and to the left are equal. Otherwise, the tip of the needle will eventually deviate from its path, and assumed shape is unstable. From conformal-mapping or numerical arguments it can be shown that, indeed, the probability for the tip of a straight needle to move forward exceeds the probability for all other deviations.

Within this picture, which so far is equivalent to a directed random walk, a small amount of noise will lead to a random distribution of kinks along the body of the needle. These kinks modify the spatial distribution of charges along the needle, and, hence, the electric field at the growing tip. A kink will enhance the probability that another kink of the same sign will be formed. That is so because it will increase the component of the field in the direction defined by the kink itself.

We can formulate this picture more precisely by assuming that the directed walk that we are considering can be approximated by a needle, and, associated with it, there is a preferred direction, which we will take to be the $X$ axis. The origin of the directed walk is at $Y=0$. Then, the $Y$ coordinate of the growing tip is determined by the number of kinks along the needle:

$$
Y(X)=\int_{0}^{X} \rho\left(X^{\prime}\right) d X^{\prime}
$$

where $\rho(X)$ is the kink density. Assuming that the charge of the kink located at $X$ is $q(X)$, the deviation of the tip from the $X$ axis will be

$$
\frac{d Y}{d X}=k \int_{0}^{X} \rho\left(X^{\prime}\right) q\left(X^{\prime}\right) \frac{Y(X)-Y\left(X^{\prime}\right)}{\left(X-X^{\prime}\right)^{2}}
$$

$k$ is a constant which, as in Eq. (3), depends in a nonuniversal way on $\eta$. These two equations determine the shape of the walk. As have been derived under the assumption that there is a preferred direction, they are only consistent if $\lim _{X \rightarrow \infty} d Y / d X=0$.

We have estimated numerically the function $q(X)$, when there are few kinks present, in an otherwise straight needle. Our results, with a high degree of accuracy, are consistent with the expression $q\left(X^{\prime}\right) \propto 1 / \sqrt{X^{\prime}\left(X-X^{\prime}\right)}$, which can be easily explained by assuming that the kinks only change the geometrical shape of the needle. We now can insert this result into Eq. (6), and check for possible shapes. The simplest assumption is a power law at large scales, $Y \sim X^{\alpha}$. Power counting shows that the only possible value of $\alpha$ is 2 . Thus, our analysis does not support the hypothesis of a straight needle with a small density of kinks. The origin of this effect can be traced back to the strong influence that the kinks near the tip exert on the direction of the walk. They affect a large amount of charge, which has a maximum at the tip. Besides, the geometrical factor which enters into Eq. (6) is also largest for a kink near the tip.

\section{CONCLUSIONS}

We have analyzed the morphology of aggregates generated with the $\eta$-rule growth law. These systems show a variety of shapes of different complexity. From a detailed study of their multifractal spectra, we infer that at least three different regimes can be distinguished.

(i) DLA patterns, with many branches at all scales, and tip splitting. They show a long tail in the high $\alpha$ side of their $f(\alpha)$ curves. They contain large regions with weak fields, where screening is important. They exist for low values of $\eta \lesssim 2$.

(ii) Branched, but dilute patterns. The difference with the previous type is that tip splitting seems to be absent. They can be found for $2 \lesssim \eta \lesssim 5$.

(iii) Thin, ribbonlike structures. They have no side branches. They are found for $\eta \gtrsim 5$. Within our accuracy, they are indistinguishable from the Laplacian random walks, discussed in the literature [6,7]. They show some multifractal features, clearly recognizable above our numerical accuracy. The $f(\alpha)$ curve, however, although nontrivial, does not show a strong dependence on the value of $\eta$.

We support the equivalence of aggregates within class (iii) and random walks with analytical arguments. We propose simple schemes for the study of these walks. Among other effects, our analysis suggests that the patterns generated tend to have a definite chirality, determined by the initial conditions. This property, along with a characteristic spiral shape at the initial stages of growth, can be appreciated in many aggregates generated with high values of $\eta$. 
We can also infer that noise is an essential ingredient in the pattern formation process. Moreover, the models considered include the parameter $\eta$ in a nonuniversal way. That is so because, in a random walk, the most relevant feature is the difference between the forward and lateral motion of the growing tip. This quantity depends on details such as the type of lattice and the width of the tip. This is an intriguing result, which implies that the fractal dimension of the Laplacian random walk need not have a universal dependence on $\eta$. Further work in this direction, as well as on the possibility of $1 / \eta$ expensions, is under way.

\section{ACKNOWLEDGMENTS}

We are thankful to O. Pla and D. Kessler for stimulating discussions. We gratefully acknowledge permission from the Advanced Computing Laboratory of Los Alamos National Laboratory and the Universidad Complutense (Spain) to use their computer facilities to grow and study the $\eta$ aggregates we deal with. A.S. was partially supported by CICyT (Spain) Project No. MAT900544 and the U.S. Department of Energy. L.M.S. is thankful to the NSF for financial support under Grant No. DMR 91-17249. F.G. and E.L. acknowledge support from CICyT (Spain).
* On leave from Instituto de Ciencia de Materiales, C.S.I.C., Cantoblanco, 28049 Madrid, Spain.

[1] T. A. Witten and L. M. Sander, Phys. Rev. Lett. 47, 1400 (1981); Phys. Rev. B 27, 5686 (1983).

[2] L. Pietronero, A. Erzan, and C. Everstz, Phys. Rev. Lett. 61, 861 (1988).

[3] T. C. Halsey and M. Leibig (unpublished).

[4] C. Amitrano, Phys. Rev. A 39, 6618 (1989).

[5] L. Niemeyer, L. Pietronero, and H. J. Wiesmann, Phys. Rev. Lett. 52, 1033 (1984); L. Pietronero and H. J. Wiesmann, J. Stat. Phys. 36, 909 (1984).

[6] J. W. Lyklema, C. Everstz, and L. Pietronero, Europhys. Lett. 2, 77 (1986).

[7] J. W. Lyklema and C. Everstz, J. Phys. A 19, L895 (1986).

[8] S. N. Majumdar, Phys. Rev. Lett. 68, 2329 (1992).

[9] C. Amitrano, A. Coniglio, and F. Di Liberto, Phys. Rev. Lett. 57, 1017; Y. Hayakawa, S. Sato, and M. Matsushita, Phys. Rev. A 36, 1963 (1987).

[10] A. Sánchez, F. Guinea, E. Louis, and V. Hakim, Physica A 191, 123 (1992).

[11] B. Derrida and V. Hakim, Phys. Rev. A 45, 8759 (1992).

[12] Y. Hayakawa, H. Kondo, and M. Matsushita, J. Phys. Soc. Jpn. 55, 2479 (1986); M. Matsushita, K. Honda, H. Toyoki, Y. Hayakawa, and H. Kondo, ibid. 55, 2618 (1986).
[13] A. Arneodo, F. Argoul, E. Bacry, J. Elezgaray, J. F. Muzy, and M. Tabard (unpublished).

[14] H. G. Hentschel and I. Procaccia, Physica 8D, 435 (1983); U. Frisch and G. Parisi, in Turbulence and Predictability in Geophysical Fluid Dynamics and Climate Dynamics, edited by M. Ghil, R. Benzi, and G. Parisi (North-Holland, Amsterdam, 1985); T. Halsey, M. H. Jensen, L. Kadanoff, I. Procaccia, and B. Shraiman, Phys. Rev. A 33, 1141 (1986); D. Bensimon, L. P. Kadanoff, S. Liang, B. I. Shraiman, and C. Tang, Rev. Mod. Phys. 58, 997 (1986); P. Meakin, Phys. Rev. A 36, 332 (1987); F. Argoul, A. Arneodo, G. Grasseau, and H. Swinney, Phys. Rev. Lett. 61, 2558 (1988); F. Argoul, A. Arneodo, J. Elezgaray, G. Grasseau, and R. Murenzi, Phys. Rev. A 41, 5537 (1990).

[15] A. B. Chhabra and R. V. Jensen, Phys. Rev. Lett. 62, 1237 (1989); A. B. Chhabra, R. V. Jensen, and K. R. Sreenivasan, Phys. Rev. A 40, 4593 (1989).

[16] P. Meakin, Phys. Rev. A 35, 2234 (1987); P. Meakin, in Phase Transitions and Critical Phenomena, edited by C. Domb and J. L. Lebowitz (Academic, London, 1988), Vol. 12, p. 336.

[17] O. Pla, F. Guinea, and E. Louis, in Growth and Form in Physics and Biology, edited by J. M. García-Ruiz, E. Louis, P. Meakin, and L. M. Sander (Plenum, New York, in press). 